\title{
Pets are irresponsible for COVID-19
}

\author{
Aijun Sun ${ }^{1}$, Youbao Zhao ${ }^{1}$, Guoqing Zhuang ${ }^{2}$, and Gaiping Zhang ${ }^{1}$ \\ ${ }^{1}$ Henan Agricultural University \\ ${ }^{2}$ Affiliation not available
}

May 26, 2020

\begin{abstract}
In late December 2019, a novel coronavirus disease (COVID-19) emerged and rapidly spread in China. Some of the pets were abandoned due to the poor economic situation, being quarantined in exotic areas or the death of their owners; these were usually adopted by an animal sanctuary. However, an extreme phenomenon of abandoning pets during the outbreak of the COVID-19. We analyzed the reasons and serious consequences of this improper behavior and gave our suggestions. We appeal for the careful protection of the pets. These innocent animals should be taken care of.
\end{abstract}

\section{Pets are irresponsible for COVID-19}

Aijun Sun, Youbao Zhao, Guoqing Zhuang*, Gaiping Zhang*

Running title: Pets are innocent

College of Animal Sciences and Veterinary Medicine, Henan Agricultural University, Zhengzhou, Henan 450002, China.

${ }^{*}$ Corresponding author.

College of Animal Sciences and Veterinary Medicine, Henan Agricultural University, Zhengzhou, Henan 450002, PR China

Email: zhuangguoqing@henau.edu.cn; zhanggaiping2003@163.com

In late January 2020, people in China had to stay at home with their pets to prevent further spread of a novel coronavirus disease (COVID-19), which is caused by the severe acute respiratory syndrome-coronavirus 2 (SARS-CoV-2) (http://www.nhc.gov.cn/xcs/yqfkdt/202001/12ec9062d5d041f38e210e8b69b6d7ef.shtml). Unfortunately, with the epidemic getting worse and the quarantine getting more restricted, some people randomly abandoned their pets due to the growing panic and anxiety induced by the misleading information about COVID-19 (http://www.myzaker.com/article/5e636add1bc8e04515000116/). We appeal for the safekeeping of the pets and taking care of them in the COVID-19 pandemic, because they are innocent and not to be blamed for any role in spreading the disease among humans.

Given the confusion made by misleading information, we try to respond to common thoughts regarding SARS-CoV-2 and pets. First, pet owners are concerned that even when taken care of their pets can be easily infected with SARS-CoV-2. In fact, pets are safe while they live with their owners under full protection and cleaning measurements. Up to date, only dogs and cats that had close contact with infected or suspected infected humans tested positive for SARS-CoV-2; in such cases, the recommended viral diagnosis of the World Organization for Animal Health (OIE) is necessary (https://www.oie.int/en/scientificexpertise/specific-information-and-recommendations/questions-and-answers-on-2019novel-coronavirus/). 
Second, pet owners are not only worried about the active infection of their pets, but also that they can be intermediate hosts of SARS-CoV-2. So far only a few wild species as potential intermediate hosts of SARS-CoV-2 have been reported, such as Malayan pangolin (Lam et al., 2020), and snakes (Ji, Wang, Zhao, Zai, \& Li, 2020). Recent serological surveys showed that the possibility of dogs and cats to be intermediate hosts is very low (Deng et al., 2020; Zhang et al., 2020, a preprint).

Furthermore, some pet owners are afraid that they might catch SARS-CoV-2 from their pets. Although the origin of SARS-CoV-2 has not yet been identified, it is clear that the major route of transmission of COVID-19 is human-to-human (Chan et al., 2020). An animal-to-human route of transmission has not been evidently reported as stated by the World Health Organization (WHO); thus, pets do not play a significant epidemiological role in the COVID-19 spread (https://www.who.int/news-room/q-a-detail/q-acoronaviruses).

Pets abandonment causes severe public health concerns in the COVID-19 pandemic. For instance, the government needs to allocate more physical, material and financial resources to take care of the increasing number of stray animals. This will put extra pressure on the integral strategy of COVID-19 prevention and control. Meanwhile, attacks by aggressive stray animals on other animals and humans will represent another public health threat.

With the rapid spread of COVID-19 worldwide, we appeal again for strict pet abandonment bans by the governments in all countries. The health and welfare of pets should be guaranteed by animal welfare and protection organizations. Information regarding COVID-19 should be critically reviewed and correctly reported to eliminate improper behaviors towards pets, which may result from misleading information spread by mass media. Last but not least, public education should include the responsibility towards pets, not just today, but every day.

\section{ACKNOWLEDGEMENTS}

This work is supported by Grants of the Starting Foundation for Outstanding Young Scientists of Henan Agricultural University (grant number 30500690).

\section{CONFLICT OF INTEREST}

The authors declare no conflict of interest relevant to this letter.

\section{ETHICAL STATEMENT}

No applicable to this comment.

\section{DATA AVAILABILITY STATEMENT}

There is no experimental data available in this letter.

\section{REFERENCES}

National Health Commission of the People's Republic of China, "Make every effort to prevent and control COVID-19"; http://www.nhc.gov.cn/xcs/yqfkdt/202001/12ec9062d5d041f38e210e8b69b6d7ef.shtml [In Chinese].

"Abandoned pets increased in COVID-19, How to deal with it?" (2020); http://www.myzaker.com/article/5e636add1bc8e04515000116/ [In Chinese].

OIE, "Questions and Answers on the 2019 Coronavirus Disease (COVID-19)" (2020); https://www.oie.int/en/scientific-expertise/specific-information-and-recommendations/questions-andanswers-on-2019novel-coronavirus/.

Lam, T. T., Shum, M. H., Zhu, H. C., Tong, Y. G., Ni, X. B., Liao, Y. S., . . . Guan, Y. (2020). Identifying SARS-CoV-2 related coronaviruses in Malayan pangolins. Nature. doi:10.1038/s41586-020-2169-0. 
Ji, W., Wang, W., Zhao, X., Zai, J., \& Li, X. (2020). Cross-species transmission of the newly identified coronavirus 2019-nCoV. J Med Virol, 92(4), 433-440. doi:10.1002/jmv.25682.

Deng, J., Jin, Y., Liu, Y., Sun, J., Hao, L., Bai, J., . . . Tian, K. (2020). Serological survey of SARS-CoV-2 for experimental, domestic, companion and wild animals excludes intermediate hosts of 35 different species of animals. Transbound Emerg Dis. doi:10.1111/tbed.13577.

Zhang. Q., Zhang, H., Huang, K., Yang, Y., Hui, X., Gao, J., ... Jin, M. (2020). SARS-CoV-2 neutralizing serum antibodies in cats: a serological investigation. BioRxiv Preprint, https://doi.org/10.1101/2020.04.01.021196.

Chan, J. F., Yuan, S., Kok, K. H., To, K. K., Chu, H., Yang, J., . . . Yuen, K. Y. (2020). A familial cluster of pneumonia associated with the 2019 novel coronavirus indicating person-to-person transmission: a study of a family cluster. Lancet, 395(10223), 514-523. doi:10.1016/S0140-6736(20)30154-9.

WHO, "Q\&A on coronaviruses (COVID-19)" (2020); https://www.who.int/news-room/q-a-detail/q-acoronaviruses. 